Western University

Scholarship@Western

6-11-2018

\title{
Changes in depression and positive mental health among youth in a healthy relationships program
}

Natalia Lapshina

The University of Western Ontario, nlapshin@uwo.ca

Claire Crooks

Western University, ccrooks@uwo.ca

Amanda Kerry

Western University

Follow this and additional works at: https://ir.lib.uwo.ca/csmh-articles

Part of the Mental and Social Health Commons, and the School Psychology Commons

\section{Citation of this paper:}

Lapshina, Natalia; Crooks, Claire; and Kerry, Amanda, "Changes in depression and positive mental health among youth in a healthy relationships program" (2018). Journal Articles. 9.

https://ir.lib.uwo.ca/csmh-articles/9 
Changes in Depression and Positive Mental Health Among Youth in a Healthy
Relationships Program
Canadian Journal of School Psychology

$1-17$

(C) The Authors 2018 Reprints and permissions: sagepub.com/journalsPermissions.nav

DOI: 10.1 I77/08295735|8777|54 journals.sagepub.com/home/cjs

@SAGE

\title{
Natalia Lapshina', Claire V. Crooks', and Amanda Kerry'
}

\begin{abstract}
Mental health promotion programming in schools and community settings is an important part of a comprehensive mental health strategy. The goal of this study was to identify and explore meaningful classes of youth based on their pre- and post-intervention depression symptoms scores with 722 youth involved in a I5-week healthy relationships and mental health promotion program. We utilized latent class growth analysis to identify depression class trajectories, controlling for group clustering effects. A threeclass solution identified high decreasing, moderate stable, and low stable trajectories. Gender, age, and reported experience of bullying victimization predicted trajectory class membership. The low stable class trajectory was associated with the highest positive mental health, followed by the moderate stable and the high decreasing trajectories. These results suggest that youth with the highest depression scores showed significant improvement in symptomatology over the course of the program.
\end{abstract}

\section{Keywords}

depression, positive mental health, mental health promotion, latent class growth analysis, adolescents

Mental health is not simply defined as the absence of mental illness, it also includes the presence of positive components. Mental health refers to a state of well-being, which allows an individual to recognize their strengths, cope with stressors, and make positive

'University of Western Ontario, London, Canada

\section{Corresponding Author:}

Natalia Lapshina, Faculty of Education, University of Western Ontario, I I 37 Western Road, Room II 54, London, Ontario, Canada N6G IG7.

Email: nlapshin@uwo.ca 
contributions to their community (World Health Organization, 2005). Consistent with this definition, Keyes operationalized a two-continua model of mental health, which states that positive mental health and mental illness are two related but different constructs, such as an individual with a mental illness may be well-functioning (Keyes, 2005). Here, positive mental health includes emotional well-being, psychological wellbeing, and social well-being (Keyes, 2002; Lamers, Westerhof, Bohlmeijer, ten Klooster, \& Keyes, 2011).

Adolescence is an important developmental period for emergence and trajectory of mental illness; therefore, this period is critical for the promotion of positive mental health (Paus, Keshavan, \& Giedd, 2008). Even when youth do not meet criteria for a diagnosable disorder, they might still experience mental health challenges that impair their functioning (Suldo, Thalji, \& Ferron, 2011). Fewer than $4 \%$ of these youth will receive services to address their needs, largely because traditional children's services sectors are relatively unable to deliver programs with the capacity and timeliness required to meet the mental health needs of young people (Leschied, Flett, \& Saklofske, 2013). Consequently, there is increasing interest in upstream work that promotes mental well-being and potentially reduces symptoms of mental health challenges among youth.

This study examined variability in changes in adolescent depression within the context of a universal healthy relationships and mental health promotion program called Fourth R Healthy Relationships Plus Program (HRPP) in a large and diverse school and community sample. In addition, the study evaluated risk factors associated with depression developmental trajectories, such as age, gender, and peer victimization in the form of cumulative bullying experiences, and examined the relationships among the identified trajectories and mental well-being.

\section{Adolescent Depression}

Adolescent depression is relatively common at both clinical and subclinical levels. In addition to diagnosable depression in adolescents which is estimated to be $4 \%$ to $8 \%$ (Garmy, Berg, \& Clausson, 2015), 29\% of youth in Grades 7 to 12 report moderately severe depressive symptoms for the past week, and $9 \%$ report severe symptoms. At 1 -year follow-up, $44 \%$ of youth with severe symptoms of depression continued to report high levels (Rushton, Forcier, \& Schectman, 2002).

Depressive symptoms development varies across gender and age, such that females are particularly vulnerable to depression in adolescence and into adulthood compared with males (E. J. Costello, Mustillo, Erkanli, Keeler, \& Angold, 2003; Twenge \& Nolen-Hoeksema, 2002). However, these broad generalizations about gender and age mask the variability within groups, and meaningful classes of youth who follow distinct developmental trajectories might exist (Nagin, 1999). Identification of distinct developmental trajectories of depression symptoms may help with prevention and diagnostics especially if different causal factors predict different trajectories (Dekker et al., 2007). For instance, studies with children and adolescents identified three to five distinct class trajectories (Musliner, Munk-Olsen, Eaton, \& Zandi, 2016). 


\section{Associated Risk Factors}

Female gender and older age predict higher depressive symptoms. A meta-analysis that examined the depression scores in youth aged 8 to 16 years revealed that in females, depression scores were relatively stable from ages 8 to 11 and then increased from ages 12 to 16. A significant gender difference was observed, beginning at age 13, with females having higher scores (Twenge \& Nolen-Hoeksema, 2002).

Furthermore, anxiety symptoms or disorders typically precede depressive symptoms and disorders (E. J. Costello et al., 2003; Zahn-Waxler, Shirtcliff, \& Marceau, 2008). For example, anxiety symptoms, such as withdrawal and isolation due to social fears, can lead to symptoms of depression including feelings of loneliness and low self-worth (Garber, 2010). Specific risk factors for depression include social anxiety, low parental support, and social skill deficits (Epkins \& Heckler, 2011).

Depression and anxiety share many core risk factors including temperament, genetics, and parental mental health; however, research suggests that these factors are not sufficient for the development of anxiety and depression. Rather, interpersonal risk and protective factors (i.e., relationships with parents and peers) moderate the core risk factors (Epkins \& Heckler, 2011). Bullying research has shown that victimization is associated with both concurrent and prospective increased odds for depression and anxiety among adolescents (Stapinski, Araya, Heron, Montgomery, \& Stallard, 2015).

In terms of latent class trajectories, females are more likely than males to follow higher depressive symptom class trajectories (D. M. Costello, Swendsen, Rose, \& Dierker, 2008). A systematic review of literature on trajectories of depressive symptoms revealed that lower academic achievement, lower family income, stressful life events, substance use, previous history of depression and anxiety, problems with peers, and other factors predicted higher depressive symptom class trajectory (Musliner et al., 2016).

\section{School-Based Mental Health Programs and Latent Class Trajectories}

Given the limitations of traditional service delivery systems, schools are an ideal setting to proactively address the mental health challenges faced by youth (Leschied et al., 2013), promote competence, and prevent unhealthy behaviours because they provide access to almost all children on a consistent basis over the formative years of development (Zenner, Herrnleben-Kurz, \& Walach, 2014). Schools remove many barriers that constrain the access of services in traditional outpatient or private settings by providing programs that are cost-effective and reach more students with fewer staff (Farmer, Burns, Phillips, Angold, \& Costello, 2003; Shonin, van Gordon, \& Griffiths, 2012).

School-based universal social-emotional learning (SEL) programs aim to prevent problems by promoting positive development and SEL competencies, including the ability to identify and manage emotions, empathize with others, build healthy relationships, and make responsible decisions (Collaborative for Academic, Social, and 
Emotional Learning, 2003). SEL programs generally have positive effects, including improvements of SEL skills, positive self-image, prosocial behaviour, and academic achievement as well as reductions in antisocial behaviours, substance use, and mental health problems (Sklad, Diekstra, De Ritter, Ben, \& Gravesteijn, 2012). SEL programs are recognized as an effective public health approach to increase mental health and well-being among children and youth (Greenberg, Domitrovich, Weissberg, \& Durlak, 2017).

\section{HRPP}

The Fourth R HRPP is an evidence-informed universal SEL program that aims to promote the development of resilience factors and equip students with the skills they need to build healthy relationships and help themselves and their peers reduce risky behaviours. It consists of 15 sessions and there is flexibility in the delivery model. The HRPP covers topics such as peer pressure, help-seeking, media literacy, healthy and unhealthy peer and dating relationships, healthy communication, the impacts of substance use, mental health, and suicide prevention (Townsley et al., 2015). There is a major focus on developing healthy and effective communication, help-seeking, and coping skills.

Even with programs designed for universal implementation, it is important to look at what works for whom. Latent class trajectories have been used to disentangle subgroups of interest. However, most of the studies that looked at mental health latent class trajectories in a context of SEL programs have focused on prevention of substance use or abuse (Kimber \& Sandell, 2009; Liu, Lynne-Landsman, Petras, Masyn, \& Ialongo, 2013), or conduct problems (Petras, Masyn, \& Ialongo, 2011; van Lier, Muthén, van der Sar, \& Crijnen, 2004; van Lier, Vuijk, \& Crijnen, 2005). Relatively few studies have examined changes in internalizing problems. For example, Spilt, Koot, and van Lier (2013) examined subgroup differences in the effectiveness of a classroom-based universal prevention program called Good Behavior Game (GBG) in Grade 1 and 2 students. They identified six subgroups that differed in baseline risk profiles and responsiveness to the GBG, such as the program prevented the development of externalizing and internalizing behaviour in children with emotional problems, low-risk children, and victimized children.

\section{The Present Study}

Given that few studies have examined latent class trajectories of internalizing problems in a context of a SEL program, it is important to identify trajectories of youth depression within the context of such programs. We address this gap in the literature by examining the trajectories of adolescent depression and associated risk factors in a sample of youth enrolled in the HRPP. We undertook this study within a realist evaluation framework in that we did not attempt to curtail the variability of participants or sites but rather sought to capture the intervention as it was implemented in real-world settings (Moore et al., 2014). 


\section{Research Questions}

Research Question 1: Is there a change in depressive symptomatology from preto post-HRPP?

Research Question 2: What are the class trajectories of depression in the context of the HRPP?

Research Question 3: How do risk factors (age, gender, cumulative bullying, and anxiety) distinguish among the depression class trajectories?

Research Question 4: How do these depression trajectories relate to mental well-being?

\section{Method}

\section{Participants}

The initial sample included 966 youth from 75 groups who participated in the HRPP program throughout the year. One community group was excluded from analyses because of an unusually wide age range (15-36 years) which affected analyses. A number of participants did not fill in post-surveys or did not provide data for measures of interest. The final sample which was based on the information from the latent growth modeling for depression included $N=722$ from 74 groups. This sample was used in all analyses to ensure consistency in results.

Participants ranged in age from 11 to 21 years $(M=15.05, S D=2.03)$; of those, $52.8 \%$ identified as female. According to the adolescent stage categories espoused by the American Academy of Pediatrics, $n=352$ (48.8\%) of our sample were in early adolescence (age 11-14 years), $n=258$ (35.7\%) were in middle (age 15-17 years), and the remaining $n=112(15.5 \%)$ were in late adolescence (age 18-21 years). The majority of participants $(n=460,64.2 \%)$ identified as White, $n=141(19.7 \%)$ Aboriginal, $n=31$ (4.3\%) Asian Canadian, $n=14(2.0 \%)$ African Canadian, $n=14$ $(2.0 \%)$ Hispanic or Latino(a), $n=7(1.0 \%)$ Arab Canadian, the rest $n=49(6.8 \%)$ identified as Other or mixed ethnicity, with $n=6(0.9 \%)$ of missing cases for ethnicity. The majority of participants $(n=284,39.3 \%)$ resided in the Province of Ontario, $n=209(28.9 \%)$ in Alberta, $n=140(19.4 \%)$ in Saskatchewan, and $n=89(12.3 \%)$ in the Northwest Territories.

\section{Materials}

Depression and anxiety. Depression (outcome) and anxiety (covariate) were measured with the Depression, Anxiety and Stress Scale (DASS-21; P. F. Lovibond \& Lovibond, 1995; S. H. Lovibond \& Lovibond, 1995). The DASS-21 is a short version of the original 42-item scale. It includes 21 self-report items assessing three negative emotion states, depression, anxiety, and stress, and has high internal reliability and adequate convergent and discriminant validity (Crawford \& Henry, 2003). Both depression and anxiety scales highly correlate with Beck Depression Inventory (BDI) and the Beck Anxiety Inventory (BAI; $r=.74$ and $r=.81$, respectively; P. F. Lovibond 
\& Lovibond, 1995). Because the scale was developed in normal and clinical populations, the obtained scores reflect a degree of depression, anxiety, and stress and, thus, cannot be used to categorize individuals into discrete diagnostic categories based on diagnostic classifications such as the Diagnostic and Statistical Manual of Mental Disorders (DSM) and International Statistical Classification of Diseases and Related Health Problems (ICD) (S. H. Lovibond \& Lovibond, 1995). However, S. H. Lovibond and Lovibond (1995) provide recommended cut-off scores for commonly used severity labels (normal, moderate, severe). To get the scores, the 21-item scores need to be doubled. For depression, the cut-off scores are as follows: normal: 0-9, mild: 10-13, moderate: $14-20$, severe: $21-27$, extremely severe: 28 and higher.

Although originally developed for adults, the scale is used in adolescent community samples (Hjemdal, Vogel, Solem, Hagen, \& Stiles, 2011; Mellor et al., 2015; Raes, Griffith, van der Gucht, \& Williams, 2014; Tully, Zajac, \& Venning, 2009; Willemsen, Markey, Declercq, \& Vanheule, 2011). In the above-mentioned adolescent samples, the reported means for depression range from $M=2.58(S D=2.87)$ to $M=4.51(S D=3.75)$ for boys, and from $M=3.90(S D=3.69)$ to $M=5.09(S D=3.99)$ for girls (Hjemdal et al., 2011; Willemsen et al., 2011), and $M=4.2(S D=4.5)$ in a combined sample (Tully et al., 2009).

Participants were asked to rate how much the statement applied to them over the past week, with answer options measured on a 4-point Likert-type scale from "did not apply to me at all" (0) to "applied to me very much or most of the time" (3). Depression was measured by seven items, such as "I felt that I had nothing to look forward to." Scores for individual items were summed up, with a total score ranging from 0 to 21 , wherein higher scores indicated higher levels of depressed mood. The subscale had high internal consistency (pretest Cronbach's $\alpha=.913$, posttest Cronbach's $\alpha=.924$ ). Seven items measured anxiety such as "I felt scared without any good reason." A total score was computed by summing up all items, ranging from 0 to 21 , with higher scores representing more anxiety. The subscale had high internal consistency at pretest (Cronbach's $\alpha=.833$ ).

Mental well-being was assessed with Mental Health Continuum-Short Form (MHC-SF; Keyes et al., 2008; Lamers et al., 2011). The MHC-SF consists of 14 items assessing emotional, social, and psychological well-being (Keyes et al., 2008). Participants reported how they were feeling in the past month, for example, "interested in life." Response options included "never" (0) to "everyday" (5). The total MHC-SF and its subscales have high internal consistency (Cronbach's $\alpha \mathrm{s}>.80$ ), test-retest reliability, and good convergent and discriminant validity in adult and adolescent samples (Keyes, 2006; Lamers et al., 2011). In the present study, individual items were summed, with an overall score ranging from 0 to 70 , and higher scores indicating better wellbeing. MHC-SF had high internal consistency (pretest Cronbach's $\alpha=.926$, posttest Cronbach's $\alpha=.955$ ).

Cumulative bullying was measured using four questions from the Bullying Evaluation and Strategies Tool (BEST; Craig et al., 2013). Participants answered questions on physical, verbal, social, and cyberbullying, for example, "Have you been bullied by other students verbally?" Response options ranged from "never" (0) to "at least once a week" (3). Due to low counts of recent incidents of bullying, responses 
were dichotomized, wherein scores 1 to 3 were coded as 1 , and reflected any instances of bullying, whereas zeros meant "never." To address nonindependence of various forms of bullying, responses were summed up, resulting in an ordinal scale ranging from 0 to 4 , where higher scores indicated more cumulative bullying experiences.

\section{Procedure}

Data were collected in 2014-2015 academic year as part of the HRPP. Prior to participant recruitment, researchers contacted school boards and community groups in four Canadian provinces: Ontario, Alberta, Northwest Territories, and Saskatchewan. Organizations interested in the project nominated individuals that agreed to participate in training and implement the program. Trainees attended a full-day in-person training program where they learned background information and specific teaching strategies for each session. Each trainee received a binder with HRPP lesson plans and support materials. The groups varied significantly in terms of geographic location, composition, and setting. There was considerable variability across settings and with respect to how youth were selected for the group. For example, in some schools, youth selfselected based on a description of the program, and in others, staff recruited youth they felt could benefit from the program. In other cases, the youth were part of a preexisting group (such as a summer camp setting). This heterogeneity is consistent with our realist approach (Moore et al., 2014) in that we were interested in how the program was implemented in various sites versus a tightly controlled approach with specific inclusion and exclusion criteria.

Parental consents were obtained for youth's participation and use of their data for research and publication. Participants filled out questionnaires assessing bullying and mental health at two time points - before the program started and after they finished the program. All data were de-identified with no personal information on file. Research Ethics approvals were obtained from the University of Western Ontario, Centre for Addiction and Mental Health, and individual school districts.

\section{Analyses}

Latent class growth analysis (LCGA). LCGA (Nagin, 1999) was used to identify meaningful classes of youth and associated changes in depression from pre-to post-participation in the program, controlling for group clustering effects. LCGA is a type of Growth Mixture Modeling (GMM) that allows the variance of latent slope and intercept to be fixed to zero. Thus, in LCGA, there is neither within-class variability nor covariance between the intercept and slope. Therefore, LCGA assumes homogeneity of individual growth within trajectories. The only parameters that vary are the intercepts and slopes between classes (Jung \& Wickrama, 2008; Nagin, 1999). Given two time points in measurement of depression, LCGA was chosen as a useful approach because it requires fewer parameters to estimate the model to avoid issues with nonconvergence and identifiability (Berlin, Parra, \& Williams, 2014). 
Model estimation started with one class, meaning one trajectory fitting all youth in the sample. Consecutively, more classes were added one by one until the best-fitting model was reached. To evaluate model fit, Bayesian information criterion (BIC), the Lo-Mendell-Rubin (LMR) test, and entropy values served as criteria. Lower BIC values and higher entropy suggested better model fit. In addition, a significant LMR test indicated that the model with a given number of classes fits better than the preceding model with one fewer class. We used mixture complex type of analyses, which allows models with both categorical and continuous latent variables and computes standard errors and a chi-square test of model fit taking into account nonindependence (clustering) of the data. Maximum likelihood robust estimator is robust to nonnormality and nonindependence of the data when used with type=complex analysis (Muthén \& Muthén, 1998-2015).

In the models, we included covariates that would distinguish among trajectory classes: participants' gender, age, and cumulative bullying as they were previously found to affect trajectories of depression (Dekker et al., 2007; Musliner et al., 2016). Due to comorbidity between anxiety and depression, the models included anxiety measured at pretest as a covariate to predict intercept of depression class trajectories.

Other analyses. After the identification of the trajectory classes, cumulative probabilities of belonging to one of the identified classes and most likely class membership were merged back into a main SPSS data file. We examined the change in positive mental health across the identified trajectory classes using two-way mixed ANOVA with post hoc follow-up tests.

\section{Results}

\section{Assumptions Testing}

All necessary assumptions for LCGA were evaluated prior to analyses. LCGA requires within-class normality; marginal distributions can be nonnormal. To address possible violations of this assumption and nonindependence of observations due to the group format delivery of the HRPP, we used maximum likelihood robust estimator which is robust to nonnormality and nonindependence of the data when used with type $=$ complex analysis (Muthén \& Muthén, 1998-2015). Assumptions of mixed-design ANOVA were satisfied.

\section{LCGA}

Using LCGA, we identified distinct classes of youth sharing a common trajectory. The three-class model fitted the data better than a two-class model, with $\mathrm{BIC}=6,726.113$, $2 \mathrm{LL} L M R=322.893, p<.0001$, and entropy $=.882$, suggesting high level of classification accuracy. The four-class model resulted in a nonsignificant LMR statistic, 2LL $\mathrm{LMR}=173.671, p=.1246$, and lower entropy value $=.846$. Table 1 summarizes 
Table I. Descriptive Statistics (M, SE) of Identified Classes of Depression.

\begin{tabular}{lcc}
\hline & Intercept & Slope \\
Trajectory class & $M(S E)$ & $M(S E)$ \\
\hline Depression & & \\
Low stable & $1.793^{* * *}$ & 0.432 \\
& $(0.135)$ & $(0.254)$ \\
Moderate stable & $9.066^{* * *}$ & -0.441 \\
& $(0.387)$ & $(0.583)$ \\
High decreasing & $17.745^{* * *}$ & $-\left.3.39\right|^{* * *}$ \\
& $(0.345)$ & $(0.702)$ \\
\hline
\end{tabular}

$* * * p<.001$.

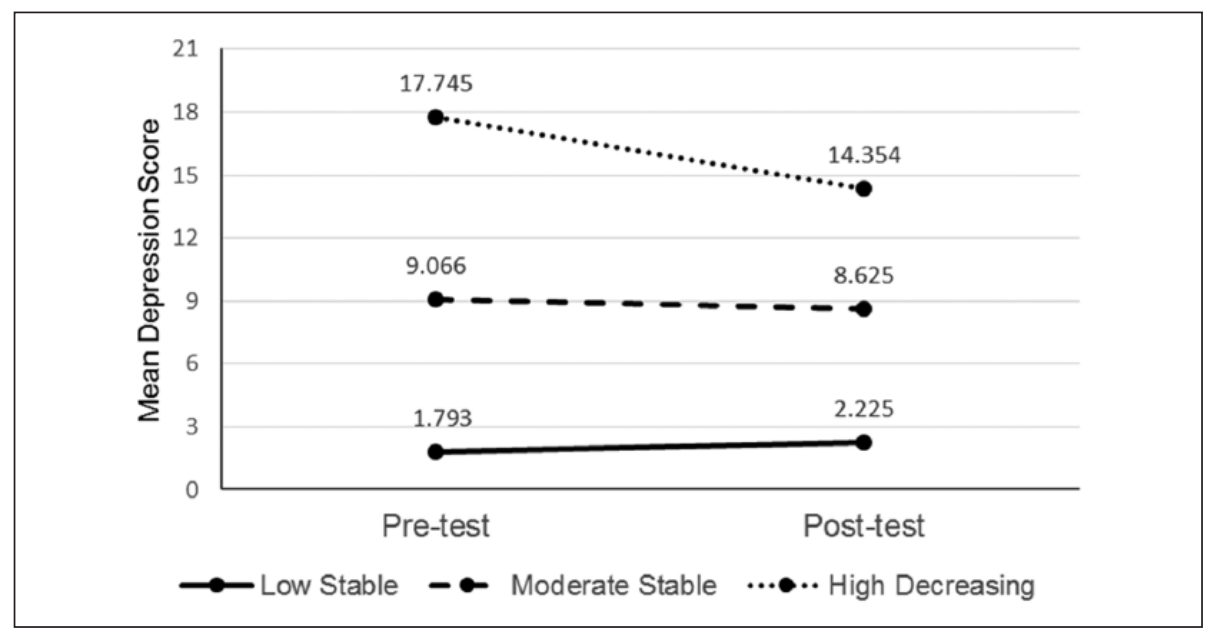

Figure I. Class trajectories of depression from pre- to post-HRPP program. Note. Lines represent class trajectories. HRPP $=$ Healthy Relationships Plus.

descriptive statistics for intercepts and slopes by trajectory class. The first class $(n=$ $370,51.25 \%$ ) represented youth with low depression, which stayed stable from pre- to post-evaluation. The second class $(n=221,30.61 \%)$ included youth with moderate depression, which remained stable over the course of the program. Finally, youth in the third class $(n=131,18.14 \%)$ reported high depression before the program start and a significant decline after the program ended. Figure 1 depicts the depression trajectories described above.

Using the guidelines for cut-off scores calculations (S. H. Lovibond \& Lovibond, 1995), the mean scores were doubled and then compared to the cut-offs; standard deviations were calculated using a formula $S D=S E \times \sqrt{n}$. The scores in the low stable trajectory could be classified as normal at pretest $(M=3.586, S D=2.597)$ and 
Table 2. Predictors of Class Membership and Related Trajectories of Depression.

\begin{tabular}{lccc}
\hline & Estimate & $S E$ & $P$ \\
\hline Moderate stable & & & \\
Gender & & 0.229 & .002 \\
Age & 0.725 & 0.066 & .024 \\
Cumulative bullying & 0.149 & 0.087 & .001 \\
Anxiety at pretest & 0.282 & 0.043 & $<.0001$ \\
High decreasing & 0.340 & & .029 \\
Gender (female) & & 0.320 & .007 \\
Age & 0.697 & 0.074 & .005 \\
Cumulative bullying & 0.200 & 0.130 & $<.0001$ \\
Anxiety at pretest & 0.364 & 0.057 & \\
\hline
\end{tabular}

Note. Higher scores for depression, anxiety, and cumulative bullying reflect higher endorsement of measured constructs.

aParametrization using low stable class as a reference.

posttest $(M=4.450, S D=4.886)$ as they are lower than 9 . The scores in the moderate stable trajectory corresponded to moderate cut-off scores as they ranged from 14 to 20 (pretest: $M=18.132, S D=5.753$; posttest: $M=17.250, S D=8.667)$. The high decreasing trajectory class reported an extremely severe depression at pretest $(M=35.49, S D$ $=3.949)$ and $M=28.708(S D=8.035)$ at posttest. Therefore, on average, participants in this trajectory class reported a decline from an extremely severe to just above the cut-off for this category.

As seen in Table 2, females and older youth were more likely to belong to moderate stable and high decreasing trajectories compared with youth in the low stable class trajectory (Class 1). Moreover, youth who reported bullying experiences and those who reported higher anxiety at pretest were more likely to belong to the moderate and high depression trajectories.

\section{Other Analyses}

Comparison of classes. After extracting the latent class trajectories in depression, class membership data were merged back into the main SPSS file. To examine whether changes in positive mental health from pre- to post-program differed as a function of depression trajectory class membership, a two-way mixed ANOVA with Bonferroni correction was conducted, wherein the within-subjects factor included pre- and postprogram MHC scores, whereas the between-subjects factor included the three depression class trajectories membership.

The two-way Time $\times$ Class interaction was not significant, $F(2,415)=0.825, p=$ $.439, \eta^{2}=.004$. The main effect of time was significant, $F(1,415)=5.132, p=.024$, $\eta^{2}=.012$. Positive mental health increased from pre-program $(M=38.752, S E=$ $0.591)$ to posttest $(M=40.203, S E=0.714)$. The main effect of depression class 
membership was also significant, $F(1,415)=188.726, p<.0001, \eta^{2}=.426$. Pairwise comparisons with Bonferroni correction revealed that all three depression trajectory classes differed from each other $(p<.0001)$. As expected, participants in the low stable class trajectory reported the highest positive mental health $(M=52.378, S E=0.709)$, followed by the moderate stable class trajectory $(M=40.642, S E=0.953)$ and the high decreasing trajectory $(M=25.411, S E=1.237)$.

\section{Discussion}

This study examined variability in changes in adolescent depression within the context of the Fourth R HRPP in a large and diverse school and community sample. In addition, the study evaluated risk factors that are associated with depression class trajectories, such as age, gender, and peer victimization.

\section{Patterns of Depression}

Depressive symptoms changed from pre- to post-HRPP, which is in line with findings of a systematic review of school-based prevention programs for depression and anxiety which found that the majority of programs are effective (Corrieri et al., 2013).

As expected, youth followed distinct class trajectories from pre- to post-program; three such trajectories were identified. The majority of youth (around 80\%) belonged to the low stable and moderate stable class trajectories with no changes in depression from pre- to post-program. Youth in the third class (almost one fifth of the sample) reported high depression at pretest and a significant decline after the program ended. The findings on developmental heterogeneity of depressive symptoms were consistent with previous research (Castelao \& Kröner-Herwig, 2013; Dekker et al., 2007; Musliner et al., 2016).

Next, we examined how risk factors (age, gender, cumulative bullying, and anxiety) distinguished between the identified depression class trajectories. The finding that females, older youth, anxious, and those who experienced multiple forms of bullying at pretest were more likely to report high depression at preprogram is consistent with other studies on risk factors and comorbidity (Dekker et al., 2007; Musliner et al., 2016). However, youth who had higher depression at pre-program followed the high decreasing trajectory. One possible explanation of the decline in depressive symptoms in this group may be related to the content of the HRPP. It might be the case that during the lessons, youth learned about healthy and unhealthy peer and dating relationships, mental health, suicide prevention, and help-seeking, and applied their knowledge and skills to their situation. Indeed, a small RCT of the same program found a reduction in bullying victimization at 12 months, which was mediated by increased intentions to seek help (Exner-Cortens, Wolfe, Crooks, \& Chiodo, manuscript in preparation). Moreover, the program's focus on relationships and interpersonal effectiveness is consistent with intervention for depression. 


\section{Mental Health}

Finally, the identified depression class trajectories were related to changes in the mental health. First, mental health increased from pre- to post-HRPP. Not surprisingly, youth in the low stable class trajectory reported the highest positive mental health, whereas those in the moderate stable class trajectory reported lower mental health, and youth in the high decreasing trajectory had the lowest mental well-being. Although expected, the differences in mental health may serve as a validation of the identified depression class trajectories. Moreover, as a strength-focused mental health promotion program, HRPP focuses on help-seeking, healthy relationships, and reduction in risky behaviours. Although, at face value, it might seem surprising that there was no interaction between class and positive mental health scores, the measure used in this study was developed to capture positive mental health as part of a dual continuum model of mental health (i.e., positive mental health and mental illness are not considered merely opposite ends of one continuum; Keyes, 2006).

\section{Limitations and Future Directions}

The present study utilized a pre-post design. Although such design informs about the amount of change in symptomatology between the two time points, it is not enough to estimate the shape of the developmental trajectory (Duncan \& Duncan, 2009). More time points are required to estimate a linear, quadratic, or other shapes of developmental trajectories. For instance, with the current design, it is impossible to determine whether youth in the high decreasing depression trajectory class continued to see improvements in their symptoms post-program. Furthermore, because this study did not have a control group, no causal conclusions can be made. Although the content of the HRPP offers a possible explanation for an improvement for youth who started highly depressed - because the program teaches about healthy relationships, symptom recognition, and help-seeking, without a comparison group these skills may not explain the observed changes in depression. At the same time, a control group was not feasible in the current context because the project was funded as a national implementation study that focused on real-world implementation in diverse settings.

Furthermore, although the accuracy of class trajectory assignment was high (88\%), it was not perfect as cases were assigned to class trajectories based on higher probability of belonging to those trajectories. Such imperfect class trajectory assignment along with posttest attrition need to be taken into account while interpreting the results.

In addition, we did not have data about other intervention or supports that youth were involved with, such as treatment for depression and/or individualized education plans. Finally, we dichotomized bullying experience because of the distribution of the data; unfortunately, this dichotomization obscures distinctions among type, severity, and chronicity of bullying experiences, which are linked to a range of mental health outcomes in this age group (e.g., Ford, King, Priest, \& Kavanagh, 2017; Thomas et al., 2016).

One future direction for better understanding the impacts of the HRPP and similar programs is to use mixed methods in understanding the impacts on positive mental 
health. The measure we used includes items that appear to measure existential purpose and global optimism, but interviews or diaries might provide more specific and concrete examples of how youth are improving their mental health based on strategies and skills that they learn in the program.

\section{Conclusion}

This study identified changes in depression from pre- to post-program in a large sample of Canadian youth who participated in the 15-week HRPP. Although this finding could in part be explained by regression to the mean, the magnitude of the change is not likely fully accounted for by that explanation. Overall, not all youth who took part in the HRPP experienced symptoms of depression in the same way over time. Instead, they followed three different trajectories from pre- to post-program. Importantly, youth with high depression reported a significant decline after the program. Among covariates that distinguished among class trajectories, anxiety at pretest strongly predicted trajectory class memberships for depression.

Although conclusive statements about program impacts of the HRPP cannot be made due to the research design for the current study, the results are nonetheless promising. That youth who begin the HRPP highly depressed show improvement over the course of the program is exciting, given the universal and low-cost nature of the program. Furthermore, positive mental health increased over the same period. These changes raise the possibility that a strengths-based, relationship-focused program can reduce negative mental health outcomes. Moving forward, there is a need to evaluate the program more rigorously, while still keeping a heterogeneous sample with respect to youth and group settings to ensure the robustness of the intervention.

\section{Acknowledgment}

We would like to thank Ray Hughes, Susan Dale, and Carrie Waters who helped to conduct this complex project.

\section{Declaration of Conflicting Interests}

The author(s) declared no potential conflicts of interest with respect to the research, authorship, and/or publication of this article.

\section{Funding}

The author(s) disclosed receipt of the following financial support for the research, authorship, and/or publication of this article: This research was supported by Health Canada. This project was funded in part by Health Canada Grant No. 1415-HQ-000081, and in part by the Public Health Agency of Canada Grant No. 1516-HQ-000027.

\section{Note}

1. Responses to stress subscale were not included as a covariate in latent class growth analysis (LCGA) because the overall model fit and findings were similar to the model 
with anxiety but the sample size dropped to $N=708$. Namely, the three-class model with stress fitted the data better than a two-class model (Bayesian information criterion $[\mathrm{BIC}]=6,567.221,2 \mathrm{LL}$ LMR $($ Lo-Mendell-Rubin $)=334.318, p<.0001$, entropy $=.887$ ), with no difference between the four- and three-class models, $2 \mathrm{LL} \mathrm{LMR}=$ $104.568, p=.136$, entropy $=.860$. The depression class trajectories were similar: low stable $\left(M_{\text {intercept }}=1.761, S E=0.137, M_{\text {slope }}=0.433, S E=0.247, p=.080\right)$; moderate stable $\left(M_{\text {intercept }}=9.058, S E=0.404, M_{\text {slope }}=-0.446, S E=0.658, p=.497\right)$; high decreasing $\left(M_{\text {intercept }}=17.733, S E=0.291, M_{\text {slope }}=-3.203, S E=0.701, p<.0001\right)$.

\section{References}

Berlin, K. S., Parra, G. R., \& Williams, N. A. (2014). An introduction to latent variable mixture modeling (part 2): Longitudinal latent class growth analysis and growth mixture models. Journal of Pediatric Psychology, 39, 188-203.

Castelao, C. F., \& Kröner-Herwig, B. (2013). Different trajectories of depressive symptoms in children and adolescents: Predictors and differences in girls and boys. Journal of Youth and Adolescence, 42, 1169-1182.

Collaborative for Academic, Social, and Emotional Learning. (2003). Safe and sound: An educational leader's guide to evidence-based social and emotional learning programs. Available from http://www.casel.org

Corrieri, S., Heider, D., Conrad, I., Blume, A., König, H. H., \& Riedel-Heller, S. G. (2013). School-based prevention programs for depression and anxiety in adolescence: A systematic review. Health Promotion International, 29, 427-441.

Costello, D. M., Swendsen, J., Rose, J. S., \& Dierker, L. C. (2008). Risk and protective factors associated with trajectories of depressed mood from adolescence to early adulthood. Journal of Consulting and Clinical Psychology, 76, 173-183.

Costello, E. J., Mustillo, S., Erkanli, A., Keeler, G., \& Angold, A. (2003). Prevalence and development of psychiatric disorders in childhood and adolescence. Archives of General Psychiatry, 60, 837-844.

Crawford, J. R., \& Henry, J. D. (2003). The Depression Anxiety Stress Scales (DASS): Normative data and latent structure in a large non-clinical sample. British Journal of Clinical Psychology, 42, 111-131.

Craig, W., Pepler, D., Hymel, S., Josephson, W., Leblanc, J., et. al (2013). PREVNet's BEST: Bullying Evaluation and Strategies Tool. Kingston, Ontario, Canada: PREVNet Inc.

Dekker, M., Ferdinand, R., van Lang, N., Bongers, I., van der Ende, J., \& Verhulst, F. (2007). Developmental trajectories of depressive symptoms from early childhood to late adolescence: Gender differences and adult outcome. Journal of Child Psychology and Psychiatry, 48, 657-666.

Duncan, T. E., \& Duncan, S. C. (2009). The ABC's of LGM: An introductory guide to latent variable growth curve modeling. Social and Personality Psychology Compass, 3, 979-991.

Epkins, C., \& Heckler, D. (2011). Integrating etiological models of social anxiety and depression in youth: Evidence for a cumulative interpersonal risk model. Clinical Child and Family Psychological Review, 1, 329-276.

Exner-Cortens, D., Wolfe, D. A., Crooks, C. V., \& Chiodo, D. (2018). A randomized controlled evaluation of a universal healthy relationships program for youth. Manuscript in preparation.

Farmer, E. M., Burns, B. J., Phillips, S. D., Angold, A., \& Costello, E. J. (2003). Pathways into and through mental health services for children and adolescents. Psychiatric Services, 54, 60-66. 
Ford, R., King, T., Priest, N., \& Kavanagh, A. (2017). Bullying and mental health and suicidal behaviour among 14- to 15-year-olds in a representative sample of Australian children. Australian \& New Zealand Journal of Psychiatry, 51, 897-908.

Garber, J. (2010). Comorbidity of anxiety and depression in youth: Implications for treatment and prevention. Clinical Psychology: Science and Practice, 17, 293-306.

Garmy, P., Berg, A., \& Clausson, E. K. (2015). A qualitative study exploring adolescents' experiences with a school-based mental health program. BMC Public Health, 15, Article 1074.

Greenberg, M. T., Domitrovich, C. E., Weissberg, R. P., \& Durlak, J. A. (2017). Social and emotional learning as a public health approach to education. The Future of Children, 27(1), 13-32.

Hjemdal, O., Vogel, P. A., Solem, S., Hagen, K., \& Stiles, T. C. (2011). The relationship between resilience and levels of anxiety, depression, and obsessive-compulsive symptoms in adolescents. Clinical Psychology \& Psychotherapy, 18, 314-321.

Jung, T., \& Wickrama, K. A. S. (2008). An introduction to latent class growth analysis and growth mixture modeling. Social and Personality Psychology Compass, 2, 302-317.

Keyes, C. L. M. (2002). The mental health continuum: From languishing to flourishing in life. Journal of Health and Social Research, 43, 207-222.

Keyes, C. L. M. (2005). Mental illness and/or mental health? Investigating axioms of the complete state model of health. Journal of Consulting and Clinical Psychology, 73(3), 539.

Keyes, C. L. M. (2006). Mental health in adolescence: Is America's youth flourishing? American Journal of Orthopsychiatry, 76, 395-402.

Keyes, C. L., Wissing, M., Potgieter, J. P., Temane, M., Kruger, A., \& Van Rooy, S. (2008). Evaluation of the mental health continuum-short form (MHC-SF) in setswana-speaking South Africans. Clinical Psychology \& Psychotherapy, 15(3), 181-192.

Kimber, B., \& Sandell, R. (2009). Prevention of substance use among adolescents through social and emotional training in school: A latent-class analysis of a five-year intervention in Sweden. Journal of Adolescence, 32, 1403-1413.

Lamers, S., Westerhof, G., Bohlmeijer, E., ten Klooster, P., \& Keyes, C. (2011). Evaluating the psychometric properties of the mental health continuum-short form (MHC-SF). Journal of Clinical Psychology, 67, 99-110.

Leschied, A., Flett, G., \& Saklofske, D. (2013). Introduction to the special issue-Renewing a vision: The critical role for schools in a new mental health strategy for children and adolescents. Canadian Journal of School Psychology, 28, 5-11.

Liu, W., Lynne-Landsman, S. D., Petras, H., Masyn, K., \& Ialongo, N. (2013). The evaluation of two first-grade preventive interventions on childhood aggression and adolescent marijuana use: A latent transition longitudinal mixture model. Prevention Science, 14, 206-217.

Lovibond, P. F., \& Lovibond, S. H. (1995). The structure of negative emotional states: Comparison of the Depression Anxiety Stress Scales (DASS) with the Beck Depression and Anxiety Inventories. Behaviour Research and Therapy, 33, 335-343.

Lovibond, S. H., \& Lovibond, P. F. (1995). Manual for the Depression Anxiety Stress Scales (2nd ed.). Sydney, Australia: Psychology Foundation.

Mellor, D., Vinet, E. V., Xu, X., Mamat, N. H. B., Richardson, B., \& Román, F. (2015). Factorial invariance of the DASS-21 among adolescents in four countries. European Journal of Psychological Assessment, 31, 138-142.

Moore, G., Audrey, S., Barker, M., Bond, L., Bonell, C., Cooper, C., . . Wight, D. (2014). Process evaluation in complex public health intervention studies: The need for guidance. Journal of Epidemiology \& Community Health, 68, 101-112. 
Musliner, K. L., Munk-Olsen, T., Eaton, W. W., \& Zandi, P. P. (2016). Heterogeneity in longterm trajectories of depressive symptoms: Patterns, predictors and outcomes. Journal of Affective Disorders, 192, 199-211.

Muthén, L.K., \& Muthén, B.O. (1998-2015). Mplus user's guide (7th ed.). Los Angeles, CA: Author.

Nagin, D. S. (1999). Analyzing developmental trajectories: A semiparametric, group-based approach. Psychological Methods, 4, 139-157.

Paus, T., Keshavan, M., \& Giedd, J. (2008). Why do many psychiatric disorders emerge during adolescence? Nature Reviews Neuroscience, 9, 947-957.

Petras, H., Masyn, K., \& Ialongo, N. (2011). The developmental impact of two first grade preventive interventions on aggressive/disruptive behavior in childhood and adolescence: An application of latent transition growth mixture modeling. Prevention Science, 12, 300-313.

Raes, F., Griffith, J. W., van der Gucht, K., \& Williams, J. M. G. (2014). School-based prevention and reduction of depression in adolescents: A cluster-randomized controlled trial of a mindfulness group program. Mindfulness, 5, 477-486.

Rushton, J., Forcier, M., \& Schectman, R. (2002). Epidemiology of depressive symptoms in the national longitudinal study of adolescent health. Journal of the American Association of Child \& Adolescent Psychiatry, 41, 199-205.

Shonin, E., van Gordon, W., \& Griffiths, M. D. (2012). The health benefits of mindfulnessbased interventions for children and adolescents. Education and Health, 30(4), 95-98.

Sklad, M., Diekstra, R., De Ritter, M., Ben, J., \& Gravesteijn, C. (2012). Effectiveness of schoolbased universal social, emotional, and behavioral programs: Do they enhance students' development in the area of skill, behavior, and adjustment? Psychology in the Schools, 49, 892-909.

Spilt, J. L., Koot, J. M., \& van Lier, P. A. (2013). For whom does it work? Subgroup differences in the effects of a school-based universal prevention program. Prevention Science, $14,479-488$.

Stapinski, L. A., Araya, R., Heron, J., Montgomery, A. A., \& Stallard, P. (2015). Peer victimization during adolescence: Concurrent and prospective impact on symptoms of depression and anxiety. Anxiety, Stress, \& Coping, 28, 105-120.

Suldo, S., Thalji, A., \& Ferron, J. (2011). Longitudinal academic outcomes predicted by early adolescents' subjective well-being, psychopathology, and mental health status yielded from a dual factor model. Journal of Positive Psychology, 6, 17-30.

Thomas, H. J., Chan, G. C., Scott, J. G., Connor, J. P., Kelly, A. B., \& Williams, J. (2016). Association of different forms of bullying victimisation with adolescents' psychological distress and reduced emotional wellbeing. Australian \& New Zealand Journal of Psychiatry, 50, 371-379.

Townsley, D., Hughes, R., Crooks, C. V., Wolfe, D. A., Burns, S., \& Kirkham, A. (2015). Healthy Relationships Plus Program: A Fourth $R$ small groups program to promote healthy relationships and positive mental health. London, Ontario, Canada: Centre for School Mental Health.

Tully, P. J., Zajac, I. T., \& Venning, A. J. (2009). The structure of anxiety and depression in a normative sample of younger and older Australian adolescents. Journal of Abnormal Child Psychology, 37, 717-726.

Twenge, J., \& Nolen-Hoeksema, S. (2002). Age, gender, race, socioeconomic status, and birth cohort differences on the Children's Depression Inventory: A meta-analysis. Journal of Abnormal Psychology, 111, 578-588. 
van Lier, P. A., Muthén, B. O., van der Sar, R. M., \& Crijnen, A. A. (2004). Preventing disruptive behavior in elementary schoolchildren: Impact of a universal classroom-based intervention. Journal of Consulting and Clinical Psychology, 72, 467-478.

van Lier, P. A., Vuijk, P., \& Crijnen, A. A. (2005). Understanding mechanisms of change in the development of antisocial behavior: The impact of a universal intervention. Journal of Abnormal Child Psychology, 33, 521-535.

Willemsen, J., Markey, S., Declercq, F., \& Vanheule, S. (2011). Negative emotionality in a large community sample of adolescents: The factor structure and measurement invariance of the short version of the Depression Anxiety Stress Scales (DASS-21). Stress \& Health, 27(3), e120-e128.

World Health Organization. (2005). Promoting mental health: Concepts, emerging evidence, practice. Geneva, Switzerland: Author.

Zahn-Waxler, C., Shirtcliff, E. A., \& Marceau, K. (2008). Disorders of childhood and adolescence: Gender and psychopathology. Annual Review of Clinical Psychology, 4, 275-303.

Zenner, C., Herrnleben-Kurz, S., \& Walach, H. (2014). Mindfulness-based interventions in schools-A systematic review and meta-analysis. Frontiers in Psychology, 5, Article 603.

\section{Author Biographies}

Natalia Lapshina is a research associate at the Centre for School Mental Health, University of Western Ontario. Her research focuses on evaluating interventions that promote the well-being of school-aged children.

Claire V. Crooks is the director of the Centre for School Mental Health at the University of Western Ontario. Her research focuses on the development and evaluation of mental health promotion and violence prevention programs. She is particularly interested in effective programming for vulnerable groups of youth.

Amanda Kerry is completing her PhD in School \& Applied Child Psychology at the University of Western Ontario. Her research and clinical interests include addressing mental health needs and preventing interpersonal violence among adolescents." 